\title{
Dosage de la cathinone et de la cathine dans des feuilles de khat (Catha edulis) saisies. Le khat pose-t-il un nouveau problème d'addiction?
}

\section{Khat (Catha edulis) : determination of cathinone and cathine concentrations in leaves of seized plants. Is khat an emerging drug concern?}

\section{RÉSUMÉ}

Originaire d'Abyssinie, où on le trouve mentionné pour la première fois au XIVime siècle, le khat et son usage masticatoire se sont répandus en Afrique de l'Est puis dans la Péninsule Arabique. Il a de nombreux synonymes : thé des abyssins, the d'Arabie, kat, kath, qat, miraa, cat, cato, catha, jimma; kus-el-salahin. Profitant de la rapidité des transports aériens, les ressortissants des pays producteurs émigrés en Europe en font l'importation illégale depuis quelques années et l'on peut craindre une extension de ce marché de niche. Poussant à l'état sauvage ou cultivé pami les plants de caféier; le khat (Catha edulis (Vahl) Forssk. ex Endl., Celastraeceae) est un grand arbuste dont les jeunes bourgeons et/ou les feuilles fraiches sont chiqués ou pris en infusion. Ses effets stimulants, proches de ceux des amphétamines, ont été très précisénent décrits par Lewin dès 1928 , et il avait classé le khat parmi les excitantia. Dans la feuille

\section{SUMMARY}

Khat and khat chewing is originated from Abyssinia, were it was first mentionned at the XIVst century. It was then introduced in East Africa and Arab Peninsula. It is described under various names : Abyssynian tea, Arabian tea, kat, kath, qat, miraa, cat, cato, catha, jimma, kus-el-salahin. With the advent of rapid transcontinental flights, migrants from East Africa and Yemen are importing illegaly khat in Europe. Khat (Catha edulis (Vahl.) Forssk. ex Endl., Celastraeceae) is an evergreen shrub which grows wild or cultivated with coffee in high valleys. Fresh khat leaves have amphetamine-like effects, and were described by L. Lewin as soon as 1928. He classified it as excitantia. In young fresh leaves, the main alkaloid is (-)-cathinone. In the dried plant and large old leaves, it is transformed in $(S, S)-(+)$-norpseudoephedrine and $(R, S)-(-)$-norephedrine. In fFrance khat and cathinone are listed as narcotics. Recent illegal imporations 
jeune et fraiche, l'alcaloïde principal est la (-)-cathinone. Dans la plante sèche et les feuilles âgées, elle est transformée en $(S, S)-(+)$-norpseudoéphédrine et $(R, S)-(-)$-noréphédrine. En France le khat lui-même et la cathinone sont inscrits sur la liste des stupéfiants. Des saisies douanières récentes de plusieurs centaines de kilogrammes de plantes fraiches nous ont permis d'effectuer de nombreux dosages. L'extraction éthanolique est suivi d'une dérivation par l'HBFA/HFIP. Le dosage est effectué par CPG-SM sur une colonne Factor Four VF-5MS, avec une programmation de température de $65^{\circ} \mathrm{C}$ à $320^{\circ} \mathrm{C}$, par rapport à des gammes de calibration préparées à partir de standards de cathine et de cathinone. Nous avons pu ainsi vérifier l'évolution rapidement décroissante de la teneur en cathinone dans les différentes parties de la plante, et que les petites feuilles des extrémités avaient des teneurs plus élevées. Comme le khat n'est pas considéré comme un stupéfiant au Royaume Uni et aux Pays Bas, on peut craindre une extension de la consommation à d'autres populations que celles des émigrés originaires d'Afrique de l'Est et de la Péninsule Arabique.

MOTS-CLÉS

Khat, cathinone, norpseudoéphédrine.

\section{Introduction}

Poussant à l'état sauvage ou cultivé parmi les plants de caféier, le khat (Catha edulis) est un grand arbuste de la famille des Celastraeceae, originaire d'Ethiopie où on le trouve mentionné pour la première fois au début du $\mathrm{XIV}^{\text {èm }}$ siècle (1). Ce n'est qu'en 1775 que sa description par l'explorateur et botaniste suédois Forsskal a été publiée en Occident $(2,3)$. Sa culture, son usage et ses effets stimulants, proches de ceux des amphétamines, ont été détaillés par Lewin dès 1928, et il avait classé le khat parmi les excitantia (1). La plante a aussi été décrite sous les noms suivants: Catha glauca, Celastrus edulis Vahl, Catha forsskalii Rich, Trigonotheca serrata Hochst, Methyscophyllum glaucum Eckl. \& Zeyher. Son nom complet actuel est : Catha edulis (Vahl) Forssk. ex Endl., Celastraceae. On lui connaît environ 80 dénominations vernaculaires, dont les plus connues sont : thé des abyssins, thé d'Arabie, thé des bushmen, kat, kath, qat, miraa, cat, cato, catha, jimma, kus-el-salahin, graba.

\section{Description botanique (2-5)}

La plante est originaire de la corne de l'Afrique, puis a été introduite au Yemen et dans la Péninsule Arabique ; on la trouve aussi dans toute l'Afrique de l'Est et en Afrique du Sud, ainsi qu'à Madagascar et en Afghanistan. La culture se pratique dans les hautes vallées froides bénéficiant d'une pluviosité suffisante ou de possibilités d'irrigation, vers $1500-2000 \mathrm{~m}$ d'altitude. Assez robuste, le khat peut pousser dans les régions were saized in Paris, and we could then analyse them. Extraction from plant material was performed by ethanol followed by a derivatization procedure with HBFA. Determination was achieved by CG-MS on a Factor Four $V F-5 M S$ with a porogrammation temperature from $65^{\circ} \mathrm{C}$ to $320^{\circ} \mathrm{C}$. Calibration curves were establihed with standard cathinone and cathine. We could verify that cathinone and cathine concentrations are decreasing dramaticaly in leaves and strands, and that the lmittle leaves at the top of the branches content more cathinone than the other parts. As $k$ hat is not banned in UK and in The Netherlands, one be aware of an increase of kaht chewing, by migrants and also by native European people.

\section{KEY-WORDS}

Khat, cathinone, norpseudoephedrine.

méditerranéennes et dans le sud des Etats-Unis d'Amérique. La taille de l'arbuste reste modeste dạns les zones arides (1-2 m), mais peut atteindre 20 à $25 \mathrm{~m}$ dans les zones tropicales. Les feuilles, lancéolées et finement dentelées, sont persistantes, d'un jaune-vert brillant qui tranche avec le rose des pédoncules et des jeunes tiges. Les éléments caractéristiques de la poudre sont bien identifiables au microscope et permettent de faire le diagnostic différentiel avec le thé (où l'on trouve des sclérites et des poils) et le maté (où les stomates sont entourés de cellules à cuticule striée) : nombreuses fibres incolores, fuselées et en groupe ; restes de canaux ; petits cristaux jumeaux d'oxalate de calcium, isolés ou en série ; fragments d'épiderme inférieur avec des stomates ; fragments d'épiderme supérieur comportant des cellules à parois finement festonnées.

\section{Mode de consommation du khat, effets}

Les extrémités des branches sont récoltées le matin et soigneusement emballées dans des feuilles de bananier ou de palmier tressées, du papier humique ou du plastique pour éviter le séchage. Ces bottes ou « mains » de 20 à 30 brindilles d'environ $500 \mathrm{~g}$ sont souvent fermées par un lien de raphia (4). On trouve également maintenant des feuilles de khat congelées et emballées sous vide, expédiées par avion en Europe et aux Etats-Unis d'Amérique (6-12) : en effet, profitant de la rapidité des transports aériens, les émigrés ressortissants des pays producteurs en font l'importation illégale depuis quelques années et l'on peut craindre une extension 
mondiale de ce marché jusqu'ici considéré comme une niche $(11,12)$.

Ce sont les feuilles fraiches de moins de 24 heures que les consommateurs arrachent une à une de chaque rameau et qu'ils mâchent longuement, en accumulant progressivement les feuilles mastiquées dans le creux de la joue. Le jus qui résulte de la mastication est avalé régulièrement. La quantité consommée au cours d'une cérémonie de khat va de 50 à $200 \mathrm{~g}$ de feuilles fraiches sur une durée d'environ 3 à 4 heures $(13,14)$.

Le khat a une nette action amphétaminique $(4,14-16)$ : anorexigène, stimulant, euphorisant, apportant une sensation subjective d'énergie accrue, de confiance en soi, d'acuité intellectuelle et facilitant la concentration et l'idéation. Tous ces effets sont fonction de l'environnement du sujet, et on peut citer Lewin (1) « le mangeur de khat est heureux d'entendre parler tour à tour ceux qui forment cercle avec lui et lui-même s'efforce de contribuer à cet entretien familier...Le khat provoque une excitation joyeuse et de la gaieté. Il éloigne le besoin de sommeil. Il redonne de l'énergie aux heures chaudes du jour, ...il empêche la faim de se faire sentir ». Au bout de quelques heures, l'excitation retombe et la séance se termine. L'adepte peut entrer dans un état de mélancolie. La léthargie peut parfois l'emporter sur l'excitation.

L'usage du khat constitue donc une pratique culturelle faisant partie intégrante de la vie sociale. Actuellement au Yemen, $90 \%$ de la population en consomme, pour $80 \%$ en 1967 (17). Les réunions sont conviviales par groupe d'une dizaine de personnes et débutent vers 3 heures de l'après-midi, ce qui fait que toute activité cesse dans le pays. Lors des mariages, les invités de marque sont honorés par du khat de qualité supérieure.

Comme la plupart des substances excitantes, le khat donne lieu à un usage excessif. Il induit une dépendance psychique modérée et on ne note pas de dépendance physique très forte $(4,11)$ : le syndrome de sevrage ne se caractérisant que par une léthargie et des cauchemars. Cependant de nombreux problèmes annexes ont été mis en évidence lors de la consommation chronique : mydriase persistante, constipation opiniâtre, tachycardie, insomnie, baisse de la libido et rarement psychose. Certains effets néfastes de l'abus sont devenus des problèmes de santé publique dans les pays de forte consommation légale : malnutrition engendrée par l'anorexie, troubles psychotiques (18), alcoolisme chronique et conduites sexuelles à risques (19), pathologies buccales (20). En Europe, des cas de conduite de véhicules sous l'influence du khat on déjà été décrits (21) : il s'agissait d'émigrés d'Afrique de l'Est.

\section{Composition chimique des feuilles de khat}

Ce sont des phénylpropylamines (khatamines) qui sont responsables de l'activité amphétaminique de la drogue $(4,6)$. Dans les feuilles fraiches et jeunes, le constituant majoritaire est la (-)-cathinone. Dans les feuilles sèches et les feuilles âgées, elle est convertie par réduction en un mélange 80-20 de (S,S)-(+)-norpseudoéphédrine (cathine) et de $(\mathrm{R}, \mathrm{S})-(-)$-noréphédrine. Les teneurs en khatamines totales sont maximales dans les jeunes pousses et dépendent de l'origine géographique, allant de 9 à $330 \mu \mathrm{g} / 100 \mathrm{mg}$. Les plantes provenant du Kenya contiennent également des homologues supérieurs en $\mathrm{C}_{6}-\mathrm{C}_{4}$ : merucathine, merucathinone, pseudomerucathine et sont plus fortement dosées en cathinone $(4,13)$. On trouve également des molécules complexes de structure variable, les cathédulines, de masse moléculaire comprise entre 600 et 1200 ; elles sont identifiées par une lettre et un chiffre $\left(\mathrm{K}_{1-5}, \mathrm{E}_{1-6}\right.$. etc. $)(4,5)$. La teneur moyenne des feuilles est de $1 \mathrm{mg}$ de cathinone, $0,9 \mathrm{mg}$ de cathine et $0,5 \mathrm{mg}$ de noréphédrine (14).

C'est cette variabilité connue des utilisateurs et déjà décrite (13-15), ainsi que les récentes saisies douanières de khat frais en région parisienne, qui nous ont amenés à rechercher et doser les khatamines dans les feuilles, et à vérifier l'évolution décroissante de la teneur en cathinone dans différentes parties de la plante.

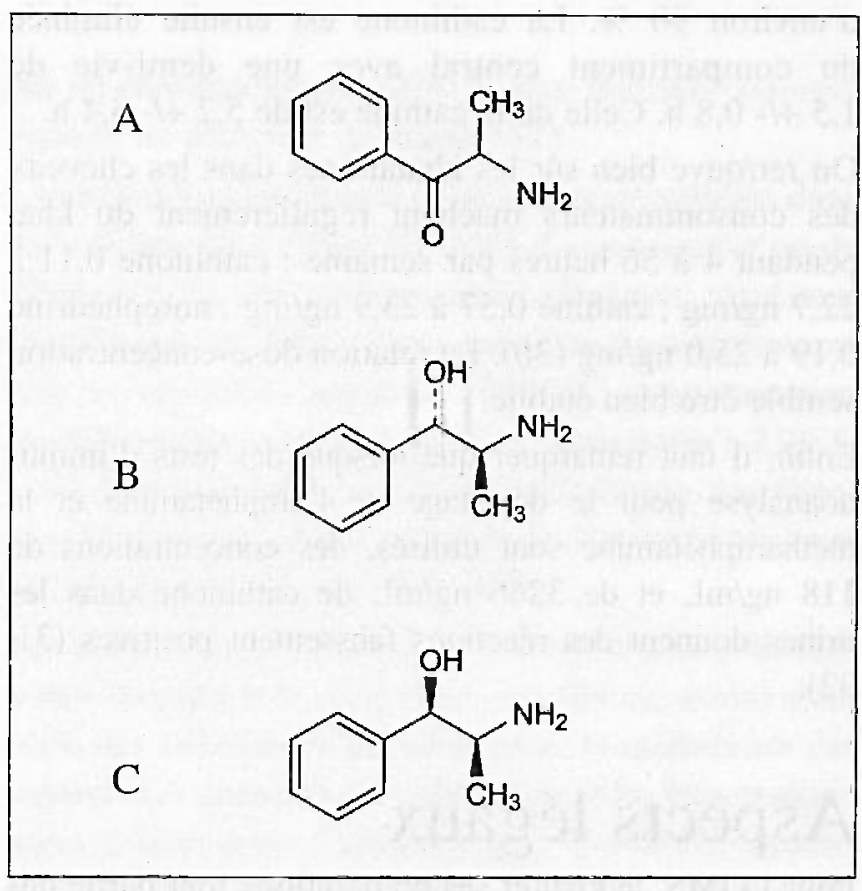

Figure 1 : Formules développées des principales khatamines: (A) cathinone ; $(B)$ cathine = norpseudoéphédrine ; $(C)$ noréphédrine. (d'après Clarke's analysis of drugs ans poison, 2004 single-user version CR-ROM). 


\section{Pharmacologie et pharma- cocinétique des khatamines}

La cathinone est considérée depuis quelques décennies comme une véritable amphétamine naturelle (15) et les méchanismes ont été bien décrits (23) : il y a relargage des catécholamines à partir des sites de stockage presynaptiques (24-26).

Les données pharmacocinétiques sont assez rares: Mathys (27) a retrouvé dans les urines, après l'ingestion de $0,5 \mathrm{mg} / \mathrm{kg}$ de (S)-(-)cathinone, de la (S)-(-) cathinone $(0,2$ à $3,8 \mu \mathrm{g} / \mathrm{mL})$ ainsi que ses métabolites la $(\mathrm{R}, \mathrm{S})$-(-)-noréphédrine $(7,2$ à $46,0 \mu \mathrm{g} / \mathrm{mL})$ et la $(\mathrm{R}, \mathrm{R})$ (-)-norpseudoéphédrine $(0,5$ à $2,5 \mu \mathrm{g} / \mathrm{mL})$.

Après une dose de $0,8 \mathrm{mg} / \mathrm{kg}$ de khat par voie orale, Wilder (28) observe un pic plasmatique de cathinone $(127+/-53 \mathrm{ng} / \mathrm{mL})$ après $127+/-30 \mathrm{~min}$. Il a déterminé que la demi-vie d'élimination de la cathinone était de 4,3+l-1,7 h. Les dosages étaient effectués par LC$\mathrm{DAD}$ et il estime que ses résultats sont peu différents de ceux de Mathys (27).

Toennes (29) a pu étudier les paramètres pharmacocinétiques des khatamines chez 4 volontaires, avec des doses de $25 \mathrm{mg}$ de cathinone, inférieures de moitié à celles habituellement utilisées dans les cérémonies de khat. Il a utilisé un modèle à deux compartiments. La muqueuse buccale est le premier site d'absorption : $59 \%$ de la cathinone et $80 \%$ de la cathine y sont absorbés. La salive et la mastication permettent une extraction optimale des principes actifs, car le rendement est d'environ $90 \%$. La cathinone est ensuite éliminée du compartiment central avec une demi-vie de $1,5+/-0,8 \mathrm{~h}$. Celle de la cathine est de $5,2+/-3,4 \mathrm{~h}$.

On retrouve bien sûr les khatamines dans les cheveux des consommateurs machant régulièrement du khat pendant 4 à 56 heures par semaine : cathinone 0,11 à $22,7 \mathrm{ng} / \mathrm{mg}$; cathine 0,57 à $23,9 \mathrm{ng} / \mathrm{mg}$; norephedrine 0,19 à $25,0 \mathrm{ng} / \mathrm{mg}$ (30). La relation dose-concentration semble être bien établie.

Enfin, il faut remarquer que lorsque des tests d'immunoanalyse pour le dépistage de l'amphétamine et la méthamphétamine sont utilisés, des concentrations de $118 \mathrm{ng} / \mathrm{mL}$ et de $3266 \mathrm{ng} / \mathrm{mL}$ de cathinone dans les urines donnent des réactions faussement positives (31, 32).

\section{Aspects légaux}

Pour I'OMS, le khat et ses préparations font partie des produits causant une dépendance depuis 1985. Mais celle-ci étant jugée facilement réversible, le khat n'est pas un souci majeur pour l'OMS en matière de stupé- fiants (5) : il n'est donc pas placé sous contrôle international.

En France, la cathinone figure sur la liste des substances classées comme stupéfiants (arrêté du 22 février 1990, JO du 7 juin 1990) et son usage est interdit selon l'article R-5179 du code de la santé publique (arrêté du 10 septembre 1992, JO du 20 septembre 1992), la cathine figure sur la liste I des substances vénéneuses (arrêté du 9 juillet 1986, JO du 26 juillet 1986) et sur la liste des substances classées comme psychotropes (arrêté du 22 février 1990, tableau III de la convention de Vienne). Le khat luimême figure sur la liste des substances classées comme stupéfiants (arrêté du 19 juillet 1995, JO du 29 juillet 1995).

En Suisse, il est inscrit dans la loi sur les stupéfiants depuis 1992. Aux Etats-Unis d'Amérique et en Afrique du Sud, il est considéré comme stupéfiant. Son usage est officiellement interdit en Arabie Saoudite, au Soudan et en Somalie. Il est toléré auYemen du Sud mais autorisé et cultivé de manière intensive au Yemen du Nord et utilisé sans aucune restriction en Ethiopie. Le khat et son usage ne sont soumis à aucune restriction au Royaume Uni et aux Pays Bas.

\section{Analyses effectuées Réactifs}

L'éthanol absolu, l'acide sulfurique et la soude $0,1 \mathrm{~N}$ sont de qualité Rectapur (VWR-Prolabo). Le dichlorométhane et l'acétate d'éthyle sont de qualité Chromanorm (VWR-Prolabo). L'anhydride heptafluorobutyrique (HFBA) et l'hexafluor-2 propanol (HFIP) proviennent respectivement de chez Interchim et de chez Sigma. Les étalons de cathinone (solution à $1 \mathrm{mg} / \mathrm{mL}$ ) et de cathine (poudre) proviennent de chez LGC Promochem.

\section{Échantillons analysés}

Les échantillons de plante ont été reçus frais, provenant d'une très importante saisie douanière $(150 \mathrm{~kg})$ effectuée quelques heures auparavant à l'aéroport de Roissy-Charles de Gaulle. La figure 2 montre quelques échantillons et des détails des branches.

Les dosages de cathine et de cathinone ont été effectués sur les petites feuilles des extrémités, sur les grandes feuilles situées plus bas et sur des fragments de tiges, selon la chronologie suivante : à J0, J1, J4, J5, J6, J7, $\mathrm{J} 8, \mathrm{~J} 11$ et $\mathrm{J} 15$, des échantillons de taille suffisante ont été placés sous vide au congélateur, le reste étant gardé à température ambiante. Après deux semaines, tous les échantillons ont été décongelés et préparés en même temps, puis analysés. 

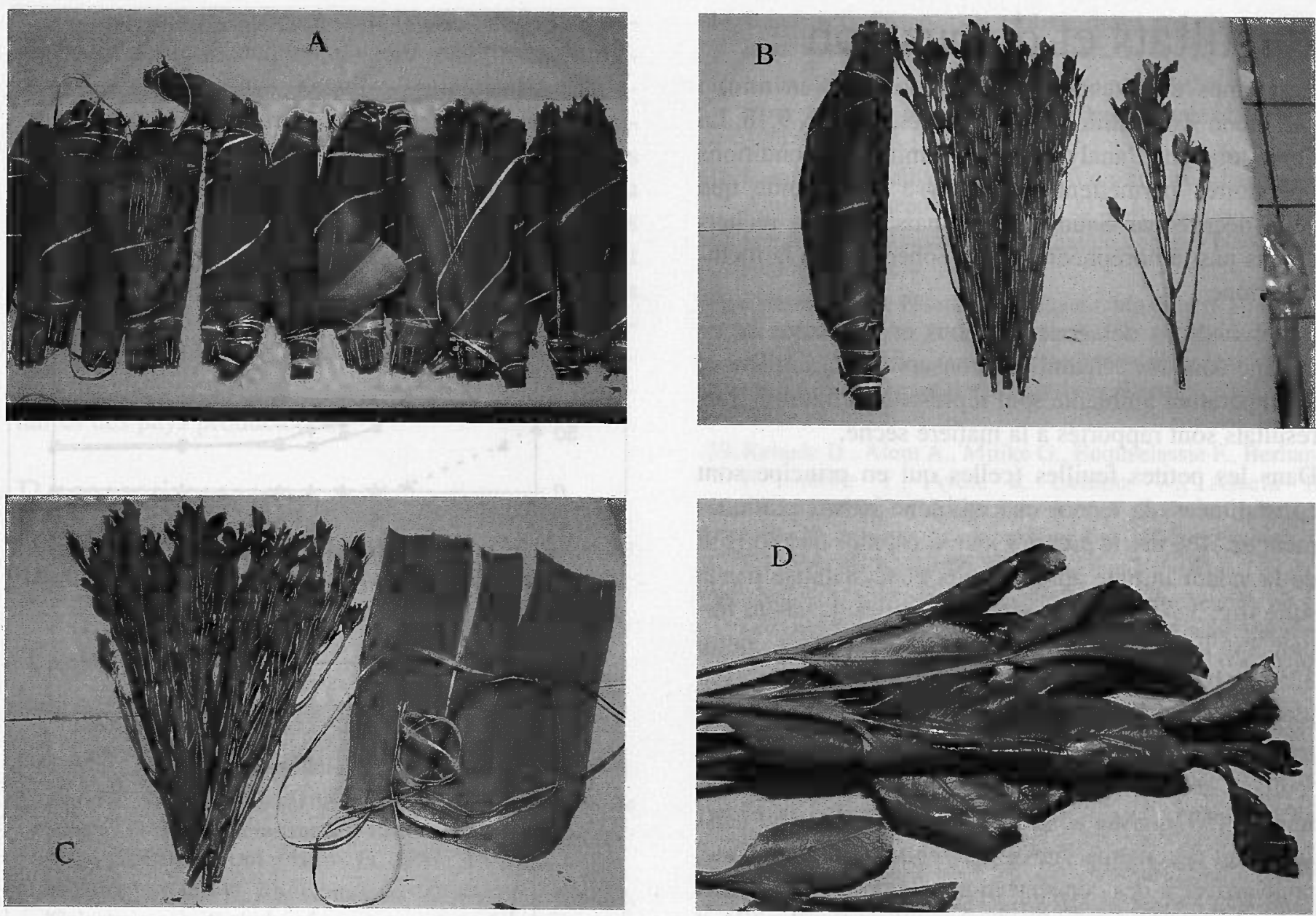

Figure 2 : Saisie de $150 \mathrm{~kg}$ de khat à Paris fin $2005:(A)$ quelques bottes; (B) et (C) détails d'une botte ; (D) extrémité d'une branchiole.

\section{Préparation et extraction}

Deux cent milligrammes de petites feuilles, de grandes feuilles et de tiges sont broyés séparément, mis en solution dans $3 \mathrm{~mL}$ d'éthanol et soumis à une sonication pendant $20 \mathrm{~min}$. Après centrifugation $5 \mathrm{~min}$ à 3500 $\mathrm{t} / \mathrm{min}$, la phase méthanolique limpide vert-olive est récupérée. A $20 \mu \mathrm{L}$ du surnageant on ajoute $20 \mathrm{ng}$ d'amphétamine- $d_{5}$ et on évapore. Le résidu sec est ensuite repris par $50 \mu \mathrm{L}$ d'acétate d'éthyle et dérivé par $50 \mu \mathrm{L}$ d'HFBA et $25 \mu \mathrm{L}$ d'HFIP pendant 30 min à $65^{\circ} \mathrm{C}$. Le résidu sec est finalement repris par $20 \mu \mathrm{L}$ d'acétate d'éthyle.

Les gammes de calibrations pour la cathinone et la cathine ont été préparées dans les mêmes conditions (0 à $1500 \mu \mathrm{g} / 100 \mathrm{mg}$ ).

\section{Dosages des khatamines}

Les dosages ont été effectuées par chromatographie en phase gazeuse couplée à la spectrométrie de masse (CPG-SM). Le système d'analyse Agilent est constitué par un chromatographe $6890 \mathrm{~N}$ avec injecteur automatique et un détecteur de masse 5973.

L'extrait dẹ́rivé est injecté ( $1 \mu \mathrm{L})$ en mode splitless dans une colonne Factor Four VF-5MS. Le gaz vecteur est de l'hélium 5.5 à une pression de $77 \mathrm{kPa}$ (avec régulateur électronique de débit). Les conditions de températures sont les suivantes : injecteur : $280^{\circ} \mathrm{C}$; programmation du four : température initiale $65^{\circ} \mathrm{C}$, puis jusqu'à $230^{\circ} \mathrm{C}$ à $20^{\circ} \mathrm{C} / \mathrm{min}$, et jusqu'à $320^{\circ} \mathrm{C}$ à $15^{\circ} \mathrm{C} / \mathrm{min}$. Le détecteur de masse est utilisé en mode impact électronique et une température de source de $300^{\circ} \mathrm{C}$.

Les molécules sont identifiées par comparaison de leur temps de rétention et de leurs spectres de masse avec ceux des substances de références, et quantifiées par rapport aux gammes de calibration. Les ions majoritaires utilisés sont : cathinone 105-77-240-169; cathine 240-169; amphétamine- $d_{5}$ 244-123. Les ions de quantification sont soulignés. 


\section{Résultats et discussion}

Les temps de rétention sont les suivants (en min) : cathinone 9,6 ; cathine 10,3 ; amphétamine- $d_{5} 9,18$. La durée totale de l'analyse est de $15 \mathrm{~min}$. Nos conditions opératoires permettent une analyse plus rapide que celle décrite par Saunier (13), mais nous ne recherchions pas la noréphédrine, ni l'éphédrine, ni la méthcathinone.

Les évolutions des concentrations en cathinone et en cathine dans les échantillons conservés à la lumière et à température ambiante sont représentées figure 3. Les résultats sont rapportés à la matière sèche.

Dans les petites feuilles (celles qui en principe sont consommées) la teneur en cathinone baisse pratiquement de $50 \%$ dès le premier jour, n'est plus que de $15 \%$ de la valeur initiale après 5 jours et se stabilise par la suite (fig. 3-A). Dans les grandes feuilles, la valeur initiale est beaucoup plus basse $(105 \mu \mathrm{g} / 100 \mathrm{mg}$ contre $325 \mu \mathrm{g} / 100 \mathrm{mg}$ ). Les concentrations résiduelles sont identiques (34-37 $\mu \mathrm{g} / 100 \mathrm{mg}$ ).

La concentration initiale de cathine dans les petites feuilles est quatre fois plus élevée que dans les grandes feuilles ( $592 \mu \mathrm{g} / \mathrm{mg}$ contre $137 \mu \mathrm{g} / 100 \mathrm{mg}$ ), et sa baisse se fait à la même vitesse que celle de la cathinone, pour arriver à des concentrations résiduelles du même ordre de grandeur dans les deux types de feuilles (74 et $40 \mu \mathrm{g} / 100 \mathrm{mg}$ respectivement) (fig. 3-B).

Dans les tiges, la teneur initiale en cathinone est faible $(57 \mu \mathrm{g} / 100 \mathrm{mg}$ ) puis devient indétectable au bout de 5 jours. En revanche, la teneur en cathine est moyenne (203 $\mathrm{\mu g} / 100 \mathrm{mg}$ ), puis augmente fortement entre le Sème et le 7ème jour, pour chuter brutalement et se stabiliser aux environs de $25 \mu \mathrm{g} / 100 \mathrm{mg}$.

Il est difficile de comparer exactement les teneurs initiales de cathinone et de cathine avec les très rares données de la littérature $(4,13,14,22)$, car aucune précision n'est donnée sur la matière analysée (fraiche ou sèche), ni sur les molécules dosées (alcalö̈des totaux ou non) ; les résultats sont parfois seulement semiquantitatifs (22).

Si la baisse rapide de la teneur en cathinone que nous avons observée est semblable aux descriptions précédentes $(4,13,22)$, nous n'avons mis en évidence la formation de cathine que dans les fragments de tige (figure 3-B). Nos résultats sont cependant du même ordre de grandeur que les données les plus précises (4), c'est-àdire une teneur en khatamines totales de l'ordre de $300 \mu \mathrm{g} / 100 \mathrm{mg}$ dans les petites feuilles le premier jour, qui baisse très rapidement de $60 \%$ en 5 jours : le khat n'est donc plus actif dès le $2^{\text {imc }}$ jour de conservation à température ambiante.

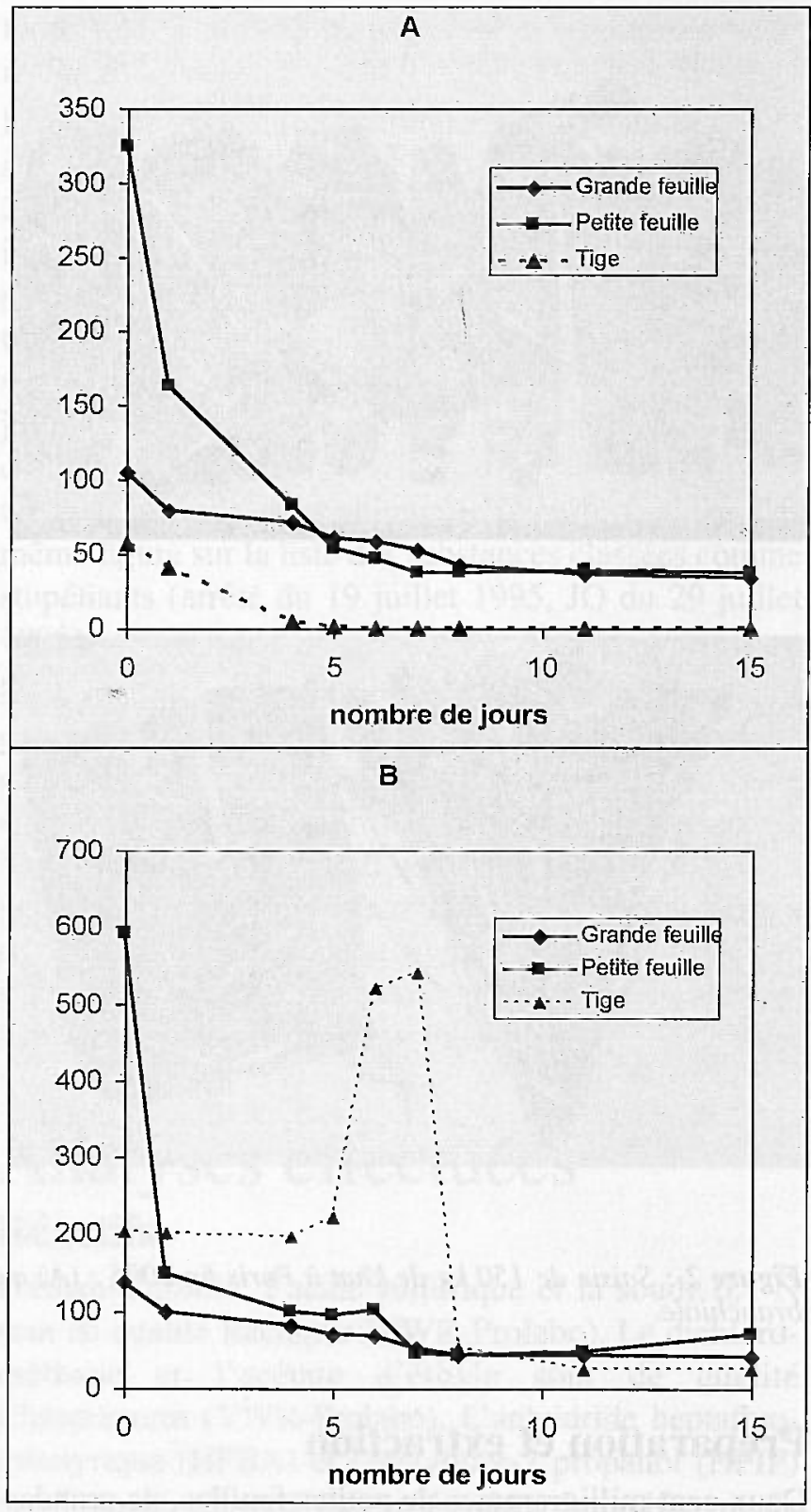

Figure 3 : Evolution des concentrations en cathinone $(A)$ et en cathine $(B)$ dans les petites feuilles, les grandes feuilles et la tige de branchioles de khat.

\section{Conclusion}

Comme l'ont noté Bruneton et Hirsch (4, 5), dans certains pays d'Afrique de l'Est et de la Péninsule Arabique l'usage du khat constitue une pratique culturelle, qui bien que remontant à l'antiquité, fait partie intégrante de la vie sociale. Malheureusement, la consommation s'est transposée maintenant en Europe et aux Etats-Unis d'Amérique, où les principaux effets pharmacologiques de la cathinone et de la cathine (coup-faim, excitation) sont recherchés. Comme la plante est cultivée dans les pays tropicaux et qu'elle se détériore très rapidement, les autorités ne s'attendent pas a priori à ce qu'elle devienne très populaire. Nous 
avons bien mis en évidence cette baisse rapide en principe actif dès le second jour de conservation à la lumière et à la température ambiante. Toutefois, les utilisateurs potentiels comptent sur la congélation et la rapidité des transports aériens pour faire des importations de produits de qualité. D'autre part il n'est pas exclu que des cultivateurs ingénieux élaborent des cultures sous serres. Enfin, des plants de Catha edulis sont maintenant dans les jardins d'ornementation, et la vente de graine sur différents sites internet se développe. Il convient donc d'être vigilant sur la consommation du khat, en principe limitée aux immigrés originaires des pays producteurs.

\section{Remerciements}

Les auteurs remercient $\mathrm{F}$. Brousse et $\mathrm{B}$. Bourrée pour leur aide technique (analyses, photographies).

\section{Références}

1. Lewin L. Les paradis artificiels, Paris, Payot, 1928 ; 290-3.

2. Paris R., Moyse H. Essai de caractérisation du kat (Catha edulis, Forssk.) (Célastracées) drogue récemment inscrite au tableau B. Ann. Pharm. Fr. 1957 ; 15 : 89-93.

3. Paris R., Moyse H. Abyssinian tea (Catha edulis Forssk, Celastraceae). A study of some samples of varying geographical origin. United Nations Office on Drugs and Crime, 1958. Disponible en ligne sur le site http://www.unodc.org, consulté le 20 décembre 2005.

4. Bruneton J. Pharmacognosie, phytochimie, plantes médicinales. Paris, Tec \& Doc, 1999 ; 883-5.

5. Hirsch C. Catha edulis, Qât (Célastracées). 2000. Disponible en ligne sur le site http://www.tela-botanica.org, consulté le 20 décembre 2005.

6. Kalix P., Brenneisen P., Koelbing U., Fisch H.U., Mathys K. Khat, a herbal drug with amphetamine properties. Schweiz. Med. Wochenschr. 1991 ; 121(43) :1561-6.

7. Anonyme. "Graba" (dried khat) seized in Kansas City. Microgram Bull. 2004 ; 37(4) : 73-4.

8. Anonyme. Khat in Northbrook, Illinois. Microgram Bull. $2004 ; 37(8): 144$.

9. Anonyme. Very large seizure of dried khat in Baltimore, Maryland. Microgram Bull. 2005 ; 38(1) : 1-2.

10. Anonyme. Fresh and dried khat in Phoenix, Arizona. Microgram Bull. 2005 ; 38(3) : 45-6.

11. Griffiths P. Gossop M., Wickenden S., Dunwoth J., Harris K., Lloyd C. A transcultural pattern of drug use: qat (khat) in the UK. Br. J. Psychiatry $1997 ; 170: 281-4$.

12. Stefan J., Mathew B. Khat chewing: an emerging drug concern in Australia? Aust. N. Z. J. Psychiatry. 2005 ; 39(9) : 842-3.

13. Saunier M., Cirimele V., Kintz P., Tracqui A., Ludes B. Dosage des alcaloïdes d'un plant de khat (Catha edulis Forsk) par CPG-SM. Toxicorama $1998 ; 10(2)$ : 95-7.
14. Baselt R.C. Disposition of toxic drugs and chemicals in man. Foster City, Biomedical Publications, 2004 ; 183-4.

15. Kalix P. The pharmacology of psychoactive alkaloids from ephedra and catha. J. Ethnopharmacol. 1991 ; 32(1-3) : 201-8.

16. Laing R.L. Hallucinogens. A forensic drug handbook. Amsterdam, Academic Press, 2003 ; 24-5.

17. Hoofer A., Osmond H. The hallucinogens, New York, Academic Poress, $1967 ; 47$.

18. Odenwald M., Neuner F., Schauer M., Elbert T., Catani C., Lingenfelder B., Hinkel H.-Häfner H., Rockstroh B. Khat use as a risk factor for psychotic disorders: a cross sectionnal and case control study in Somalia. BMC Med. $2005 ; 3(1): 5$

19. Kebede D., Alem A., Mitike G,, Enquselassie F., Berhane F., Abebe Y., Ayele R. Lemma W., Assefa T., Gebremichael T. Khat and alcohol use and risky sex behavior among in-school and out-of-school youth in Ethiopia. BMC Public Health $2005 ; 5: 109$.

20. Al-Hebshi N.N., Skaug N. Effect of khat chewing on 14 selected periodontal bacteria in sub-and supragingival plaque of a young male population. Oral Microbiol. Immunol. $2005 ; 20(3)$ : 141-6.

21. Toennes S.W., Kauert G.F. Driving under the influence of khat-alkaloid concentrations and observation in forensic cases. Forensic Sci. Int. 2004 ; 140(1) : 85-90.

22. Guantai A.N., Maitai C.K. Relative distribution of cathinone and D-norpseudoephedrine in Catha edulis (Miraa) growing in Kenya. East Afr. Med. J. 1982 ; 59(6) : 394-8.

23. Kalix P. Cathinone, a natural amphetamine. Pharmacol. Toxicol. $1992 ; 70(2): 77-86$.

24. Kalix P. Catha edulis, a plant that has amphetamine effects. Pharm. World Sci. $1996 ; 18(2)$ : 69-73.

25. Patel N.B. Mechanism of action of cathinone, the active ingredient of khat (Catha edulis). East Afr. Med. J. $2000 ; 77(6): 329-32$.

26. Al-Hebshi N.N., Skaug N. Khat (Catha edulis)-an uptated review. Addict. Biol. $2005 ; 10(4)$ : 299-307.

27. Mathys K. Brenneisen R. Determination of cathinone and its metabolites norephedrine and norpseudoephedrine in urine by HPLC-DAD. J. Chromatogr. 1992 ; 593(1-2) : 79-85.

28. Wilder P., Mathys K., Brenneisen R., Kalix P., Fisch H.U. Pharmacodynamics and pharmacokinetics of khat: a controlled study. Clin. Pharmacol. Ther. 1994 ; 55(5) : 556-62.

29. Toennes S.W., Harder S. Schramm M., Niess C., Kauert G.F. Pharmacokinetics of cathinone, cathine, and norephedrine after the chewing of khat leaves. $\mathrm{Br} \mathrm{J}$. Clin. Pharmacol. 2003 ; 56(1) : 125-30.

30. Sporkert F., Pragst F., Bachus R., Masuhr F., Harms L. Determination of cathinone, cathine and norephedrine in hair of Yemenite khat chewer. Forensic Sci. Int. 2003; $133(1-2): 39-46$.

31. Paul B.D., Cole K.A. Cathinone (Khat) and methcathinone (CAT) in urine specimens: a GC-MS detection procedüre. J. Anal. Toxicol. $2001 ; 25(7)$ : 525-30.

32. Toennes S.W., Kauert G.F. Excretion and detection of cathinone, cathine, and phenylpropanolamine in urine after khat chewing. Clin. Chem. 2002 ; 48(10) : 1715-9. 